\title{
Successful External Cephalic Version: Factors Predicting Vaginal Birth
}

\author{
Pei Shan Lim, ${ }^{1}$ Beng Kwang Ng, ${ }_{1}^{1}$ Anizah Ali, ${ }^{2}$ Mohamad Nasir Shafiee, \\ Nirmala Chandralega Kampan, ${ }^{1}$ Nor Azlin Mohamed Ismail, ${ }^{1}$ \\ Mohd Hashim Omar, ${ }^{1}$ and Zaleha Abdullah Mahdy ${ }^{1}$ \\ ${ }^{1}$ Department of Obstetrics and Gynaecology, UKM Medical Centre, Malaysia \\ ${ }^{2}$ Department of Obstetrics and Gynaecology, UPM, Malaysia
}

Correspondence should be addressed to Pei Shan Lim; peishan9900@yahoo.com

Received 27 August 2013; Accepted 4 November 2013; Published 22 January 2014

Academic Editors: H. Sheng and M. C. Vallejo

Copyright (C) 2014 Pei Shan Lim et al. This is an open access article distributed under the Creative Commons Attribution License, which permits unrestricted use, distribution, and reproduction in any medium, provided the original work is properly cited.

Purpose. To determine the maternal and fetal outcomes of successful external cephalic version (ECV) as well as factors predicting vaginal birth. Methods. The ECV data over a period of three years at Universiti Kebangsaan Malaysia Medical Centre (UKMMC) between 1 September 2008 and 30 September 2010 was reviewed. Sixty-seven patients who had successful ECV were studied and reviewed for maternal, fetal, and labour outcomes. The control group comprised patients with cephalic singletons of matching parity who delivered following the index cases. Results. The mean gestational age at ECV was $263 \pm 6.52$ days (37.5 weeks \pm 6.52 days). Spontaneous labour and transient cardiotocographic (CTG) changes were the commonest early adverse effects following ECV. The reversion rate was $7.46 \%$. The mean gestational age at delivery of the two groups was significantly different $(P=0.000)$ with $277.9 \pm$ 8.91 days and $269.9 \pm 9.68$ days in the study group and control groups, respectively. The study group needed significantly more inductions of labour. They required more operative deliveries, had more blood loss at delivery, a higher incidence of meconiumstained liquor, and more cord around the neck. Previous flexed breeches had a threefold increase in caesarean section rate compared to previous extended breeches ( $44.1 \%$ versus $15.2 \%, P=0.010)$. On the contrary, an amniotic fluid index (AFI) of 13 or more is significantly associated with a higher rate of vaginal birth $(86.8 \%$ versus $48.3 \%, P=0.001)$. Conclusions. Patients with successful ECV were at higher risk of carrying the pregnancy beyond 40 weeks and needing induction of labour, with a higher rate of caesarean section and higher rates of obstetrics complications. Extended breech and AFI 13 or more were significantly more likely to deliver vaginally postsuccessful ECV. This additional information may be useful to caution a patient with breech that ECV does not bring them to behave exactly like a normal cephalic, so that they have more realistic expectations. However, these predictive factors needed further confirmation and hopefully, in the future, they would be able to further enhance counselling prior to ECV.

\section{Introduction}

Publication of the International Randomized Term Breech Trial in 2000 [1] appeared to confirm the presumption that primary caesarean section would reduce morbidity and mortality among children in breech presentation at term. It brought about a major change in clinical practice worldwide. The Term Breech Trial, however, had a major impact on the attitude of obstetricians towards vaginal breech delivery [2] as the number of caesarean sections for breech presentation at term increased considerably over the following years and is still on the rise. This increased rate of caesarean section might accelerate the associated maternal morbidity related to anaesthesia or operative interventions. It also placed subsequent pregnancies and labour at a higher risk.

In order to control this rise in caesarean section rate obstetricians seriously looked into external cephalic version (ECV) as a method that can safely reduce the incidence of breech presentation at term [3, 4]. Both the American College of Obstetricians and Gynaecologists (ACOG) and British 
TABLE 1: Fetal characteristic at external cephalic version (ECV).

\begin{tabular}{lc}
\hline & $n=67$ \\
\hline $\begin{array}{l}\text { Sonographic EFW at ECV (kg) } \\
\text { Type of breech }\end{array}$ & $2.87 \pm 0.29$ \\
$\quad$ Flexed, sum (\%) & $34(50.7)$ \\
Extended, sum (\%) & $33(49.3)$ \\
AFI (cm) & $13.77 \pm 2.45$ \\
AFI (sum, \%) & \\
$10.0-12.0$ & $23(34.3)$ \\
$12.1-16.0$ & $31(46.3)$ \\
$16.1-18.0$ & $9(13.4)$ \\
$>18.0$ & $4(6.0)$ \\
Placenta (sum, \%) & \\
Anterior & $22(32.8)$ \\
Posterior & $34(50.7)$ \\
Fundal & $11(16.5)$ \\
\hline
\end{tabular}

All expressed in mean \pm SD unless specified. EFW: estimated fetal weight.

Royal College of Obstetricians and Gynaecologists (RCOG) recommended the use of ECV as first line management of breech presentations at term $[5,6]$.

Although the sole intent of ECV was to reduce the overall caesarean sections for breech presentation, there is still overwhelming controversy as to whether this aim is ultimately achieved [7]. To date, there are conflicting evidences, as some studies had shown an increased rate of caesarean delivery even after a successful ECV [8], whereas others did not [911]. Some studies also quoted an increase in instrumental and overall obstetrics intervention following a successful ECV [8]. Therefore, this 3-year audit aimed to concentrate our review on the maternal, fetal, and labour outcomes following successful ECV at Universiti Kebangsaan Malaysia Medical Centre (UKMMC). We also aimed to explore the factors predicting successful vaginal birth following ECV.

\section{Materials and Methods}

2.1. Study Design. A retrospective review was carried out on ECV performed over a period of three years between 1 September 2008 and 30 September 2010 at the Department of Obstetrics and Gynaecology, UKMMC. Patient details and outcomes of ECV were obtained from the ECV record book. Case notes for those who had successful ECV were reviewed for maternal, fetal, and labour outcomes. Successful ECV was defined as cephalic presentation at the end of ECV. All successful ECV were reviewed after one week as per hospital protocol to exclude spontaneous reversion. In this review, the study group comprised patients with successful ECV who delivered in UKMMC. Patients lost to follow-up after ECV were excluded from the study.

It is our departmental policy to offer ECV routinely, in the absence of contraindications, to women with breech presentation after 36 weeks. Following a successful version, the management of labour and delivery did not differ from other patients. They were allowed vaginal birth unless subsequent maternal or fetal indications arise necessitating a change in the mode of delivery. Therefore, the antenatal and intrapartum care of patients following successful ECV was governed by the same departmental protocol as for other pregnancies. Induction of labour was performed at 40 weeks plus 10 days of gestation in the absence of any other problems.

Data regarding fetal presentation at the onset of labour, mode of delivery, intra-partum and postpartum events, and neonatal outcome were recorded. Adverse outcome or events including those occurring within 24 hours of ECV were recorded. Adverse outcomes subsequent to this period were also considered; however, these would be more difficult to attribute to the ECV. Intra-partum events and details were documented. Neonatal assessment was performed after delivery. Other additional information pertaining to the ECV success as well as rates of reversion was also collected and analysed.

The controls were the next patients who delivered a normal cephalic singleton with similar (matched) parity. Information was obtained from the labour room delivery record book.

Data collection, storage, and analysis were performed using the Statistical Package for Social Sciences (SPSS) for Windows version 13.0 software. Student's $t$-test was used to compare continuous data and chi-square and Fisher's exact test for categorical data. Statistical significance was set at the $5 \%$ level (95\% confidence interval).

\section{Result}

There were 22, 25, and 20 cases of successful ECV in 2008, 2009 , and 2010, respectively. Hence the total number of ECV performed successfully in this 3-year period was 73. Six cases were lost to follow-up: four delivered in other hospitals and two were untraceable. Therefore, only 67 cases were included in the analysis.

3.1. The Successful ECV Group. The success rate of ECV was $51.4 \%$ (73/142 cases) over the three years. ECV was performed at a mean gestational age of $263 \pm 6.52$ days (37.5 weeks \pm 6.52 days). The mean sonographic estimated fetal weight at the time of ECV was $2.87 \pm 0.29 \mathrm{~kg}$ (Table 1). There were 34 (50.7\%) flexed breeches and 33 (49.3\%) extended breeches. The mean amniotic fluid index (AFI) was $13.77 \pm 2.45 \mathrm{~cm}$ at ECV with range of 12.1 to 16.0 (46.3\%). Posterior placentation $(50.7 \%)$ was the commonest compared to anterior placentation (32.8\%) and fundal placentation (16.4\%) (Table 1).

There were five deliveries within 24 hours following successful ECV. Three of them had successful vaginal delivery, while one parturient had an emergency caesarean section for poor progress of labour (Table 2). As for fetal complications, four fetuses had CTG changes following ECV. Three were transient changes and only one had persistent pathological CTG changes. The transient CTG changes were, namely, fetal bradycardia and late decelerations. The CTG subsequently reverted spontaneously back to reassuring traces. The persistently pathological CTG was persistent fetal bradycardia 
TABLE 2: Complications occurring within 24 hours of external cephalic version (ECV).

\begin{tabular}{lccc}
\hline \multicolumn{1}{c}{ Maternal complications } & & \multicolumn{2}{c}{ Fetal complications } \\
\hline Uterine rupture & 0 & Transient CTG changes & $3(4.5 \%)$ \\
PROM & 0 & Pathological CTG changes & $1(1.5 \%)$ \\
Abruptio placenta & 0 & Fetal death within 24 hours of ECV & 0 \\
Labour & $4(6.0 \%)$ & Fetal death $>24$ hours after ECV & 0 \\
Emergency LSCS & $2(3.0 \%)$ & Others & 0 \\
\hline
\end{tabular}

PROM: Prelabour rupture of membrane.

LSCS: Lower segment caesarean section.

TABLE 3: Maternal characteristics of the study group (successful external cephalic version) and control group (spontaneous cephalic presentation).

\begin{tabular}{|c|c|c|c|}
\hline & Study group $(n=67)$ & Control group $(n=67)$ & $P$ value \\
\hline Age (years) & $29.91 \pm 4.53$ & $28.84 \pm 4.79$ & 0.184 \\
\hline Parity & $0.96 \pm 1.22$ & $0.94 \pm 1.22$ & \\
\hline Primigravida (\%) & $33(49.3 \%)$ & $33(49.3 \%)$ & \\
\hline Para $1-4(\%)$ & $32(47.8 \%)$ & $32(47.8 \%)$ & (Matched variable) \\
\hline Para $\geq 5(\%)$ & $2(3.0 \%)$ & $2(3.0 \%)$ & \\
\hline \multicolumn{4}{|l|}{ Ethnicity } \\
\hline Malays (\%) & $46(68.7 \%)$ & $50(74.6)$ & \multirow{4}{*}{$\chi^{2}=3.033, P=0.387$} \\
\hline Chinese (\%) & $14(20.9 \%)$ & $7(10.4 \%)$ & \\
\hline Indian (\%) & $2(3.0 \%)$ & $3(4.5 \%)$ & \\
\hline Others (\%) & $5(7.4 \%)$ & $7(10.5 \%)$ & \\
\hline Gestational age at ECV (days) & $263.0 \pm 6.52$ & NA & NA \\
\hline Gestational age at delivery (days) & $277.9 \pm 8.91$ & $269.9 \pm 9.68$ & 0.000 \\
\hline BMI at delivery $\left(\mathrm{kg} / \mathrm{m}^{2}\right)$ & $26.32 \pm 4.42$ & $23.26 \pm 3.68$ & 0.000 \\
\hline Interval of ECV to delivery (median, quartile) (days) & $15.0(8.0,21.0)$ & NA & NA \\
\hline
\end{tabular}

All expressed in mean \pm SD unless specified.

warranting emergency caesarean section. The baby was born $2.84 \mathrm{~kg}$ with a low cord $\mathrm{pH}$ of 7.115 requiring neonatal intensive care unit (NICU) admission for respiratory distress (Table 2). The baby was discharged well after 3 days.

Out of the 67 cases of successful ECV, five (7.46\%) fetuses reverted back to either breech presentation or transverse. All of them presented in labour, between 9 and 24 days after ECV, and had emergency caesarean delivery.

3.2. The Successful ECV Group versus the Control Group. A total of sixty-seven parity-matched controls were compared with the ECV group. The maternal age ranged between 19 and 45 years in the ECV group and between 20 and 42 years in the control group, giving rise to no significant difference in the mean age $(29.91 \pm 4.53$ versus $28.84 \pm 4.79, P=0.184)$ in both groups (Table 3 ).

Gestational age at delivery was significantly higher in the ECV group compared to the control group (277.9 \pm 8.91 days versus $269.9 \pm 9.68$ days, $P=0.000)$. The ECV group also had a significantly higher body mass index (BMI) (26.32 \pm $4.42 \mathrm{~kg} / \mathrm{m}^{2}$ versus $\left.23.26 \pm 3.68 \mathrm{~kg} / \mathrm{m}^{2}, P=0.000\right)$ compared to the control group (Table 3 ).

A large number of subjects in the ECV group required induction of labour as compared to the control group $(31.3 \%$ versus $4.5 \%, P=0.000$ ) (Table 4 ). Most patients in the control group (95.5\%) came in spontaneous labour. The presence of meconium-stained liquor was significantly higher (nearly fold) in the ECV group in comparison to the control group (26.9\% versus $3.0 \%, P=0.000)$. Cord around neck was seen fairly commonly in the ECV group but none was observed in the control group ( $13.4 \%$ versus $0 \%, P=0.000$ ).

The rate of successful spontaneous vertex delivery was higher in the control group compared to the study group (71.6\% versus $\left.59.7 \%, \chi^{2}=2.119, P=0.203\right)$. Similarly, there were more vaginal births (combined spontaneous vertex delivery and instrumental delivery) in the control group compared to the ECV group (80.6\% versus $70.1 \%, \chi^{2}=1.970$, $P=0.229$ ). However, these differences were not statistically significant. Obstetrics intervention, that is, combined instrumental delivery and caesarean section, was more frequently observed in the ECV group compared to the control group ( $40.3 \%$ versus $28.4 \%, \chi^{2}=2.119, P=0.203$ ), but again the difference was not statistically significant. Median blood loss at delivery was higher in the ECV group $(300 \mathrm{~mL}$ versus $250 \mathrm{~mL}, Z=-1.811, P=0.70$ ), but it was not statistically significant. A number of subjects in the study group had postpartum haemorrhage $\left(9.0 \%\right.$ versus $3.0 \%, \chi^{2}=2.127$, $P=0.274)$. However, the difference was not statistically significant (Table 4). There were no significant differences in birth weights, Apgar scores, cord $\mathrm{pH}$ values, and gender 
TABLE 4: Maternal labour outcome.

\begin{tabular}{|c|c|c|c|}
\hline & Study group $(n=67)$ & Control group $(n=67)$ & $P$ value \\
\hline \multicolumn{4}{|l|}{ Mode of delivery (sum, \%) } \\
\hline Vaginal delivery & $40(59.7)$ & $48(71.6)$ & \multirow{3}{*}{-} \\
\hline Instrumental delivery & $7(10.4)$ & $6(9.0)$ & \\
\hline Caesarean section & $20(29.9)$ & $13(19.4)$ & \\
\hline \multicolumn{4}{|l|}{ Type of labour (sum, \%) } \\
\hline Induction & $21(31.3)$ & $3(4.5)$ & \multirow{2}{*}{$\begin{aligned} \chi^{2} & =16.445 \\
P & =0.000\end{aligned}$} \\
\hline Spontaneous & $46(68.7)$ & $64(95.5)$ & \\
\hline Estimated blood loss (median, mL) & $300.0(250.0,300.0)$ & $250.0(250.0,300.0)$ & $\begin{array}{l}Z=-1.811 \\
P=0.70\end{array}$ \\
\hline Postpartum haemorrhage (sum, \%) & $6(9.0)$ & $2(3.0)$ & $\begin{aligned} \chi^{2} & =2.127 \\
P & =0.274\end{aligned}$ \\
\hline $\begin{array}{l}\text { Obstetrics intervention (instrumental delivery and caesarean } \\
\text { section) (sum, \%) }\end{array}$ & $27(40.3)$ & $19(28.4)$ & $\begin{aligned} \chi^{2} & =2.119 \\
P & =0.203\end{aligned}$ \\
\hline
\end{tabular}

TABLE 5: Fetal outcome.

\begin{tabular}{|c|c|c|c|}
\hline & Study group $(n=67)$ & Control group $(n=67)$ & $P$ value \\
\hline Birth weight (mean $\pm \mathrm{SD}, \mathrm{kg}$ ) & $3.183 \pm 0.377$ & $3.075 \pm 0.45$ & $P=0.137$ \\
\hline \multicolumn{4}{|l|}{ Apgar score (median) } \\
\hline 1 minute & 8 & 9 & $\begin{array}{c}Z=-1.560 \\
P=0.119\end{array}$ \\
\hline 5 minutes & 9 & 9 & $\begin{array}{c}Z=-1.482 \\
P=0.138\end{array}$ \\
\hline Apgar score 5 minutes $<7$ & 0 & 0 & \\
\hline \multicolumn{4}{|l|}{ Cord $\mathrm{pH}$} \\
\hline Mean \pm SD & $7.296 \pm 0.079$ & $7.309 \pm 0.079$ & $P=0.300$ \\
\hline$<7.2($ sum,$\%)$ & $8(11.9)$ & $6(9.0)$ & $\begin{array}{r}\chi^{2}=0.319 \\
P=0.572\end{array}$ \\
\hline \multicolumn{4}{|l|}{ Gender (sum, \%) } \\
\hline Male & $32(47.8)$ & $31(46.3)$ & \multirow{2}{*}{$\begin{aligned} \chi^{2} & =0.030 \\
P & =0.863\end{aligned}$} \\
\hline Female & $35(52.2)$ & $36(53.7)$ & \\
\hline Presence of meconium (sum, \%) & $18(26.9)$ & $2(3.0)$ & $\begin{array}{c}\chi^{2}=15.046 \\
P=0.000\end{array}$ \\
\hline Cord abnormality (sum, \%) & $9(13.4)$ & 0 & $\begin{aligned} \chi^{2} & =9.648 \\
P & =0.003\end{aligned}$ \\
\hline NICU admission (sum, \%) & $3(4.5)$ & $2(3.0)$ & $\begin{aligned} \chi^{2} & =0.208 \\
P & =1.000\end{aligned}$ \\
\hline
\end{tabular}

NICU: neonatal intensive care unit.

distribution between the two groups. The number of babies admitted to NICU was similar between the two groups $(4.5 \%$ versus $3.0 \%, P>0.05$ ) (Table 5).

3.3. Factors Influencing Mode of Delivery in the ECV Group. Among patients in the ECV group who went post-dates (beyond 40 weeks), two-thirds had successful vaginal delivery while a third required caesarean section (Table 6). The rate of caesarean section was higher amongst nulliparae (39.4\% versus $20.6 \%)$ and those with BMI $<25 \mathrm{~kg} / \mathrm{m}^{2}(35.5 \%$ versus $25.0 \%$ ). Women with anterior placentation had the highest caesarean section rate of $40.9 \%$ as compared to $36.4 \%$ in women with fundal placentation and $20.6 \%$ in women with posterior placentation. Previous flexed breeches had almost a threefold increased risk of caesarean section compared to previous extended breeches $(44.1 \%$ versus $15.2 \%, P=0.010)$. On the contrary, AFI of thirteen or more is significantly associated with higher rates of vaginal birth $(86.8 \%$ versus $48.3 \%, P=0.001)$. Both sonographically estimated fetal weight and birth weight did not predict the mode of delivery. This may be due to fetal macrosomia being an exclusion factor in selecting patients for ECV. Male babies had a twofold higher caesarean section rate compared to female babies (40.6\% versus $20.0 \%$ ), but this was not statistically significant. Among all the above-tabulated factors, only extended breech and AFI $\geq 13$ at ECV were found to be statistically significant in predicting a successful vaginal birth (Table 6). 
TABLE 6: Factors predicting successful vaginal delivery following successful external cephalic version.

\begin{tabular}{|c|c|c|c|}
\hline & Vaginal + instrumental delivery $(n=47)$ & Caesarean section $(n=20)$ & $P$ value \\
\hline Age (years) & $29.57 \pm 4.26$ & $30.70 \pm 5.14$ & 0.356 \\
\hline Gestation at ECV (days) & $263.13 \pm 6.12$ & $262.85 \pm 7.54$ & 0.875 \\
\hline \multicolumn{4}{|l|}{ Gestation at delivery } \\
\hline$\leq 40$ weeks/ $\leq 280$ days & $30(73.1 \%)$ & $11(26.9 \%)$ & \multirow{2}{*}{$\begin{aligned} \chi^{2} & =0.461 \\
P & =0.587\end{aligned}$} \\
\hline$>40$ weeks/>280 days & $17(65.4 \%)$ & $9(34.6 \%)$ & \\
\hline Interval from ECV to delivery (days) & $14.34 \pm 8.54$ & $16.20 \pm 9.71$ & 0.436 \\
\hline \multicolumn{4}{|l|}{ Parity } \\
\hline Nulliparous & $20(60.6 \%)$ & $13(39.4 \%)$ & \multirow{2}{*}{$\begin{aligned} \chi^{2} & =2.828 \\
P & =0.093\end{aligned}$} \\
\hline Multiparous & $27(79.4 \%)$ & $7(20.6 \%)$ & \\
\hline \multicolumn{4}{|l|}{ BMI } \\
\hline$<25 \mathrm{~kg} / \mathrm{m}^{2}$ & $20(64.5 \%)$ & $11(35.5 \%)$ & \multirow{2}{*}{$\begin{aligned} \chi^{2} & =0.874 \\
P & =0.350\end{aligned}$} \\
\hline$\geq 25 \mathrm{~kg} / \mathrm{m}^{2}$ & $27(75.0 \%)$ & $9(25.0 \%)$ & \\
\hline \multicolumn{4}{|l|}{ Placenta } \\
\hline Anterior & $13(59.1 \%)$ & $9(40.9 \%)$ & \multirow{3}{*}{$P=0.509$} \\
\hline Posterior & $27(79.4 \%)$ & $7(20.6 \%)$ & \\
\hline Fundal & $7(63.6 \%)$ & $4(36.4 \%)$ & \\
\hline \multicolumn{4}{|l|}{ Type of breech } \\
\hline Flexed & $19(55.9 \%)$ & $15(44.1 \%)$ & \multirow{2}{*}{$P=\mathbf{0 . 0 1 0}$} \\
\hline Extended & $28(84.8 \%)$ & $5(15.2 \%)$ & \\
\hline AFI $(\mathrm{cm})$ & $14.15 \pm 2.39$ & $12.89 \pm 2.44$ & $P=0.054$ \\
\hline \multicolumn{4}{|l|}{$\mathrm{AFI}(\mathrm{cm})$} \\
\hline$<13$ & $14(48.3)$ & $15(51.7)$ & \multirow{2}{*}{$\begin{aligned} \chi^{2} & =11.683 \\
P & =0.001\end{aligned}$} \\
\hline 13 and $>13$ & $33(86.8)$ & $5(13.2)$ & \\
\hline \multicolumn{4}{|l|}{ Sonographic EFW } \\
\hline$<3.0 \mathrm{~kg}$ & $34(70.8 \%)$ & $14(29.2 \%)$ & \multirow{2}{*}{$\begin{aligned} \chi^{2} & =0.038 \\
P & =0.846\end{aligned}$} \\
\hline$\geq 3.0 \mathrm{~kg}$ & $13(68.4 \%)$ & $6(31.6 \%)$ & \\
\hline \multicolumn{4}{|l|}{ Birth weight } \\
\hline$<3.0 \mathrm{~kg}$ & $16(80.0 \%)$ & $4(20.0 \%)$ & \multirow{2}{*}{$\begin{aligned} \chi^{2} & =1.321 \\
P & =0.250\end{aligned}$} \\
\hline$\geq 3.0 \mathrm{~kg}$ & $31(66.0 \%)$ & $16(34.0 \%)$ & \\
\hline \multicolumn{4}{|l|}{ Gender } \\
\hline Male & $19(59.4 \%)$ & $13(40.6 \%)$ & \multirow{2}{*}{$\begin{aligned} \chi^{2} & =3.396 \\
P & =0.065\end{aligned}$} \\
\hline Female & $28(80.0 \%)$ & $7(20.0 \%)$ & \\
\hline
\end{tabular}

All quantitative data expressed in mean \pm SD. EFW: Estimated Fetal Weight.

\section{Discussion}

Clinical audit is an effective quality improvement process to evaluate important clinical issues [12]. Among the popular topics of concern and controversy in obstetrics practice is the management of breech presentation at term. Breech presentation has become an outstanding issue in the past decade due to its marked contribution to the rising caesarean section (CS) rate, especially as a repercussion of the Term Breech Trial. This retrospective review aimed to analyse the outcome following successful ECV in our hospital. The success rate of ECV over these three years was $51.4 \%$, which has improved compared to those reported in previous local studies $[13,14]$. Hofmeyr et al. demonstrated a similar success rate of ECV $(40-50 \%)$ in routine clinical practice [15]. Conversely, Lau and Ben-Arie et al. reported higher success rates of $69.5 \%$ and $78.7 \%[16,17]$.

Amongst the 67 women with successful ECV, the commonest complications occurring within 24 hours of ECV were spontaneous labour $(6.0 \%)$ and transient CTG changes $(4.5 \%)$. This is similar to the report by Lau et al. in which transient CTG changes occurred at a rate of $3.3 \%$ in their series of 243 cases of ECV [16]. The median interval between ECV and delivery was 15.0 days $[8,18]$. Out of the 67 subjects with successful ECV, five fetuses reverted to either breech or transverse (7.46\%). This was slightly higher in comparison to other studies where Lau and Feyi-Waboso et al. reported reversion rates of only $4.1 \%$ and $3.0 \%$, respectively $[16,19]$. The possibility of higher reversion rate could be due to an undiagnosed unstable lie prior to ECV as three of these five 
cases reverted to an abnormal lie, which required caesarean delivery.

Significantly, more subjects in the ECV group required induction of labour compared to the control group. This was attributable to a larger number of subjects in the ECV group who went beyond 40 weeks (280 days) of gestation, thus increasing the rate of induction of labour. Chan et al. reported a higher rate of induction of labour amongst those with successful ECV but did not show any difference in the rate of prolonged pregnancy [8]. It can easily be postulated that the higher prevalence of prolonged pregnancy with successful ECV is contributed by delayed stretching of the lower uterine segment by the delay in descent of the head into the pelvis. Obviously, the fetal head stretches the lower segment better than the breech. One of the factors determining the length of pregnancy is neurological behaviour of the fetus. Kean et al. suggested that there are neurological differences between term breech fetuses and cephalic fetuses [20]. They demonstrated a significant difference in the number of fetal behaviour state transitions between breech and cephalic fetuses. Although breech fetuses demonstrated similar total length of time in their movements, the sustained movements were shorter. However, the actual impact of this difference remained unclear. They suggested that the failure to sustain activity might be related to underlying neurodevelopmental problem.

Median blood loss at delivery and the prevalence of postpartum haemorrhage were both higher in the ECV group compared to the control group although the differences were statistically insignificant. This may be explained by the more frequent obstetrics interventions and operative delivery in the ECV group.

It was widely recognised that breech presentation was associated with a higher perinatal mortality and morbidity regardless of the mode of delivery. This was supported by Danielian et al. [21], who concluded that breech presentation was a signal for potential fetal handicap. A few studies based in Nigeria documented low 5-minute Apgar scores and unacceptably high perinatal mortality rates in association with vaginal breech deliveries $[22,23]$. Our study documented no significant difference between the ECV group and the control group in terms of birth weight, gender, 1- and 5-minute Apgar scores, cord $\mathrm{pH}$ values, or rate of admission to the neonatal intensive care unit. However, meconium staining of liquor was found to be nearly ninefold higher amongst the ECV group in comparison to the control group. Again, this may be attributable to a higher prevalence of postdatism in the ECV group $(P=0.000)$. Cord abnormalities were not an uncommon finding amongst the ECV group but none was seen in the control group.

In attempting to identify factors predicting a successful vaginal birth after ECV, it was found that only extended breech and AFI $\geq 13$ were statistically significant in this role. This finding is in agreement with that of Zandstra and Mertens who reported that the success rate of ECV in extended breech was higher compared to flexed breech (34.6\% versus 14.1\%, $P<0.05$ ) [24]. In our study, flexed breech contributed slightly higher to successful ECV compared to extended breech (50.7 versus 49.3\%); however,
post-ECV extended breech favours successful vaginal birth better than flexed breech (44.1\% versus 15.2\%) (Table 6).

Male fetuses are known to have an increased risk of fetal distress during labour [18] and instrumental as well as caesarean deliveries $[25,26]$. This agrees with our finding of male fetuses being more likely to be delivered via CS following successful ECV compared to female (40.6\% versus $20 \%$ ) although the difference was not statistically significant. Possible explanations include differences in intra-partum cardiotocographic changes, higher prevalence of placental dysfunction, and differences in energy metabolism between male and female fetuses [27-29].

The majority of the caesarean deliveries following successful ECV were for fetal distress and labour dystocia (35.0\% each). In the control group, $30.7 \%$ of caesarean section was for fetal distress and only $15.4 \%$ was for labour dystocia. This finding was consistent with results reported by Lau et al. in which there were 2.5- and 2.8-fold increases in intra-partum fetal distress and labour dystocia following ECV [16]. The increased caesarean section and instrumental delivery for fetal distress may be attributed to obstetrician bias, as they are likely to practise a lower threshold for both caesarean section and instrumental delivery for those with successful ECV. Chan et al. [8]; however, did not agree with this explanation. They suggested that fetuses with breech presentation were less tolerant to the stress of labour compared to cephalic fetuses. As for labour dystocia, Vézina et al. [9] proposed a few hypotheses in an attempt to explain the increased incidence of labour dystocia amongst parturients following successful ECV. The proposed hypotheses were as follows: (1) uterine anomalies or atypical maternal pelvis configuration may cause both an increased risk for fetal breech presentation and a higher risk of dystocia during labour, (2) in patients who had a successful ECV, because of the need to have a mobile fetus, it was more likely to have a fetus with an unmoulded, unengaged head or a head in an asynclitic position or a combination of those factors during labour, and (3) patients with successful ECV may have an increased uterine compliance that, in time, can be associated with abnormal uterine contractility resulting in labour dystocia [9].

\section{Limitations}

This was a retrospective review, with 6 patients lost to followup. A similar but prospective study with a larger sample size should be conducted in the future to ascertain the findings of this study. As for the estimated blood loss, no standard quantification technique was applied, leading to the possibility of bias.

\section{Conclusion}

Patients with successful ECV were at higher risk of carrying the pregnancy beyond 40 weeks of gestation needing induction of labour. Previous extended breech and AFI 13 or more were significant contributors towards successful vaginal birth following ECV. This additional information may be useful to caution a patient with breech that ECV does not bring them 
to behave exactly like a normal cephalic, so that they have more realistic expectations. However, these predictive factors needed further confirmation and hopefully, in the future, they would be able to further enhance our counselling prior to ECV.

\section{Ethical Approval}

This study was approved by UKM Research Ethics Committee (UKMREC) and given the project code FF-379-2011.

\section{Disclosure}

The study was presented as oral presentation at the 1st O\&G Scientific Meeting at Hotel Equatorial, Bangi, Malaysia, on 1516 April 2011.

\section{Conflict of Interests}

The authors declare that there is no conflict of interests regarding the publication of this paper.

\section{Authors' Contribution}

Professor Dr. Zaleha Abdullah Mahdy mooted the idea of the ECV clinic and subsequently set it up with the help of Professor Dr. Nor Azlin Mohamed Ismail. Associate Professor Dr. Pei Shan Lim was responsible for the study design and contributed to the data analysis and final writing of this study. Dr. Beng Kwang Ng was responsible for writing up the first draft and correcting it after comments by the other authors. All coauthors contributed to proof reading and literature review.

\section{Acknowledgment}

The authors wish to thank the staff at the ECV clinic.

\section{References}

[1] M. E. Hannah, W. J. Hannah, S. A. Hewson, E. D. Hodnett, S. Saigal, and A. R. Willan, "Planned caesarean section versus planned vaginal birth for breech presentation at term: a randomised multicentre trial," The Lancet, vol. 356, no. 9239, pp. 1375-1383, 2000.

[2] E. K. Hutton, M. E. Hannah, and J. Barrett, "Use of external cephalic version for breech pregnancy and mode of delivery for breech and twin pregnancy: a survey of Canadian practitioners," Journal of Obstetrics and Gynaecology Canada, vol. 24, no. 10, pp. 804-810, 2002.

[3] G. J. Hofmeyr and R. Kulier, "External cephalic version for breech presentation at term," Cochrane Database of Systematic Reviews, no. 2, Article ID CD000083, 2000.

[4] R. J. Collaris and S. G. Oei, "External cephalic version: a safe procedure? a systematic review of version-related risks," Acta Obstetricia et Gynecologica Scandinavica, vol. 83, no. 6, pp. 511$518,2004$.
[5] Royal College of Obstetricians and Gynaecologists, The management of breech presentation. Guideline No. 20. RCOG Clinical Green Top Guidelines, 2001.

[6] Committee on Obstetric Practice, "ACOG committee opinion. Mode of term singleton breech delivery. No. 265, December 2001. American College of Obstetricians and Gynecologists," International Journal of Gynecology \& Obstetrics, vol. 77, pp. 6566, 2002.

[7] C. Clock, J. Kurtzman, J. White, and J. H. Chung, "Cesarean risk after successful external cephalic version: a matched, retrospective analysis," Journal of Perinatology, vol. 29, no. 2, pp. 96-100, 2009.

[8] L. Y.-S. Chan, T. Y. Leung, W. Y. Fok, L. W. Chan, and T. K. Lau, "High incidence of obstetric interventions after successful external cephalic version," An International Journal of Obstetrics and Gynaecology, vol. 109, no. 6, pp. 627-631, 2002.

[9] Y. Vézina, E. Bujold, J. Varin, G. P. Marquette, and M. Boucher, "Cesarean delivery after successful external cephalic version of breech presentation at term: a comparative study," American Journal of Obstetrics and Gynecology, vol. 190, no. 3, pp. 763768, 2004.

[10] A. Ben-Haroush, T. Perri, J. Bar et al., "Mode of delivery following successful external cephalic version," American Journal of Perinatology, vol. 19, no. 7, pp. 355-360, 2002.

[11] J. R. Wax, K. Sutula, T. Lerer, J. D. Steinfeld, and C. J. Ingardia, "Labor and delivery following successful external cephalic version," American Journal of Perinatology, vol. 17, no. 4, pp. 183186, 2000.

[12] D. Siassakos, H. Anderson, and K. Panter, "Breech presentation: an audit project as means of pursuing clinical excellence," Journal of Obstetrics and Gynaecology, vol. 25, no. 7, pp. 642647, 2005.

[13] M. I. Nor Azlin, H. Haliza, Z. A. Mahdy, I. Anson, M. N. Fahya, and M. A. Jamil, "Tocolysis in term breech external cephalic version," International Journal of Gynecology and Obstetrics, vol. 88, no. 1, pp. 5-8, 2005.

[14] N. A. Mohamed Ismail, M. Ibrahim, N. Mohd Naim, Z. A. Mahdy, M. A. Jamil, and Z. R. Mohd Razi, "Nifedipine versus terbutaline for tocolysis in external cephalic version," International Journal of Gynecology and Obstetrics, vol. 102, no. 3, pp. 263-266, 2008.

[15] G. J. Hofmeyr and R. Kulier, "External cephalic version for breech presentation at term," Cochrane Database of Systematic Reviews, no. 2, Article ID CD000083, 2000.

[16] T. K. Lau, K. W. Kit, and M. Rogers, "Pregnancy outcome after successful external cephalic version for breech presentation at term," American Journal of Obstetrics and Gynecology, vol. 176, no. 1 I, pp. 218-223, 1997.

[17] A. Ben-Arie, S. Kogan, M. Schachter, Z. J. Hagay, and V. Insler, "The impact of external cephalic version on the rate of vaginal and cesarean breech deliveries: a 3-year cumulative experience," European Journal of Obstetrics Gynecology and Reproductive Biology, vol. 63, no. 2, pp. 125-129, 1995.

[18] D. J. Bekedam, S. Engelsbel, B. W. J. Mol, S. E. Buitendijk, and K. M. Van der Pal-de Bruin, "Male predominance in fetal distress during labor," American Journal of Obstetrics and Gynecology, vol. 187, no. 6, pp. 1605-1607, 2002.

[19] P. A. Feyi-Waboso, C. O. Selo-Ojeme, and D. O. Selo-Ojeme, "External cephalic version (ECV): experience in a sub-Saharan African hospital," Journal of Obstetrics and Gynaecology, vol. 26, no. 4, pp. 317-320, 2006. 
[20] L. H. Kean, C. Suwanrath, S. S. Gargari, D. S. Sahota, and D. K. James, "A comparison of fetal behaviour in breech and cephalic presentations at term," British Journal of Obstetrics and Gynaecology, vol. 106, no. 11, pp. 1209-1213, 1999.

[21] P. J. Danielian, J. Wang, and M. H. Hall, "Long term outcome by method of delivery of fetuses in breech presentation at term: population based follow up," British Medical Journal, vol. 312, no. 7044, pp. 1451-1453, 1996.

[22] O. A. Ilesanmi, O. A. Sobowale, and O. A. Marinho, "Outcome of 441 breech singleton deliveries at the Catholic Hospital, Oluyoro, Ibadan," African Journal of Medicine and Medical Sciences, vol. 25, no. 1, pp. 41-46, 1996.

[23] E. O. Orji and K. O. Ajenifuja, "Planned vaginal delivery versus caesarean section for breech presentation in Ile-Ife, Nigeria," East African Medical Journal, vol. 80, no. 11, pp. 589-591, 2003.

[24] H. Zandstra and H. J. M. M. Mertens, "Improving external cephalic version for foetal breech presentation," Facts, Views \& Vision in ObGyn, vol. 5, no. 2, pp. 85-90, 2013.

[25] E. Sheiner, A. Levy, M. Katz, R. Hershkovitz, E. Leron, and M. Mazor, "Gender does matter in perinatal medicine," Fetal Diagnosis and Therapy, vol. 19, no. 4, pp. 366-369, 2004.

[26] E. Lieberman, J. M. Lang, A. P. Cohen, J. Frigoletto F.D., D. Acker, and R. Rao, "The association of fetal sex with the rate of cesarean section," American Journal of Obstetrics and Gynecology, vol. 176, no. 3, pp. 667-671, 1997.

[27] N. W. Dawes, G. S. Dawes, M. Moulden, and C. W. G. Redman, "Fetal heart rate patterns in term labor vary with sex, gestational age, epidural analgesia, and fetal weight," American Journal of Obstetrics and Gynecology, vol. 180, no. 1, pp. 181-187, 1999.

[28] A. Edwards, A. Megens, M. Peek, and E. M. Wallace, "Sexual origins of placental dysfunction," The Lancet, vol. 355, no. 9199, pp. 203-204, 2000.

[29] C. A. Clarke and U. Mittwoch, "Changes in the male to female ratio at different stages of life," British Journal of Obstetrics and Gynaecology, vol. 102, no. 9, pp. 677-679, 1995. 




The Scientific World Journal
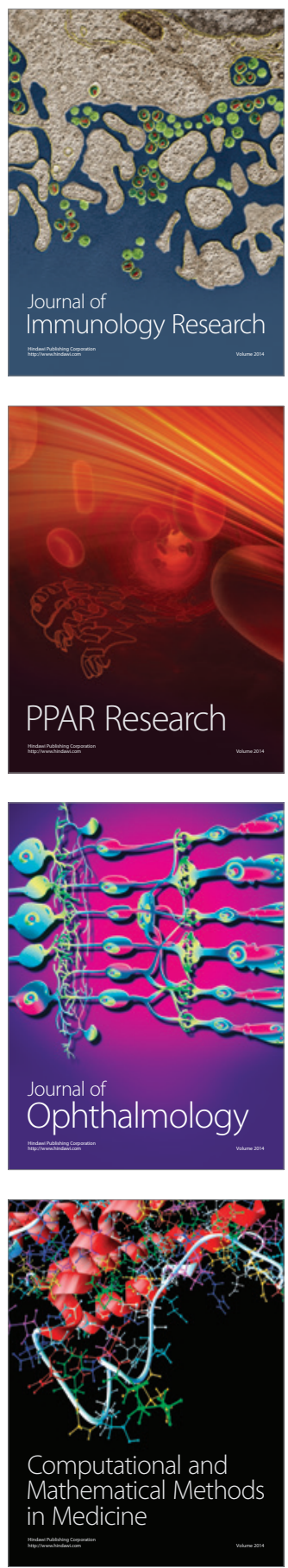

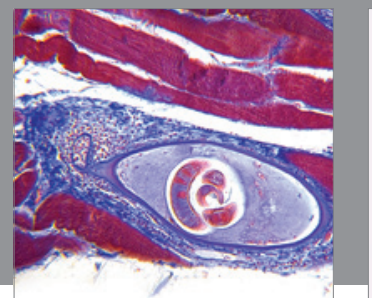

Gastroenterology

Research and Practice
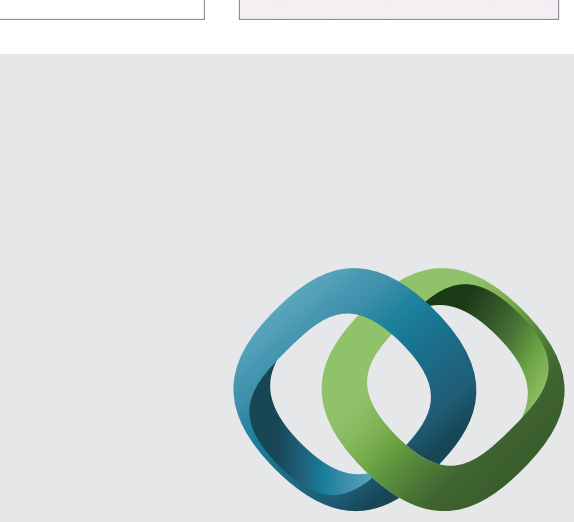

\section{Hindawi}

Submit your manuscripts at

http://www.hindawi.com
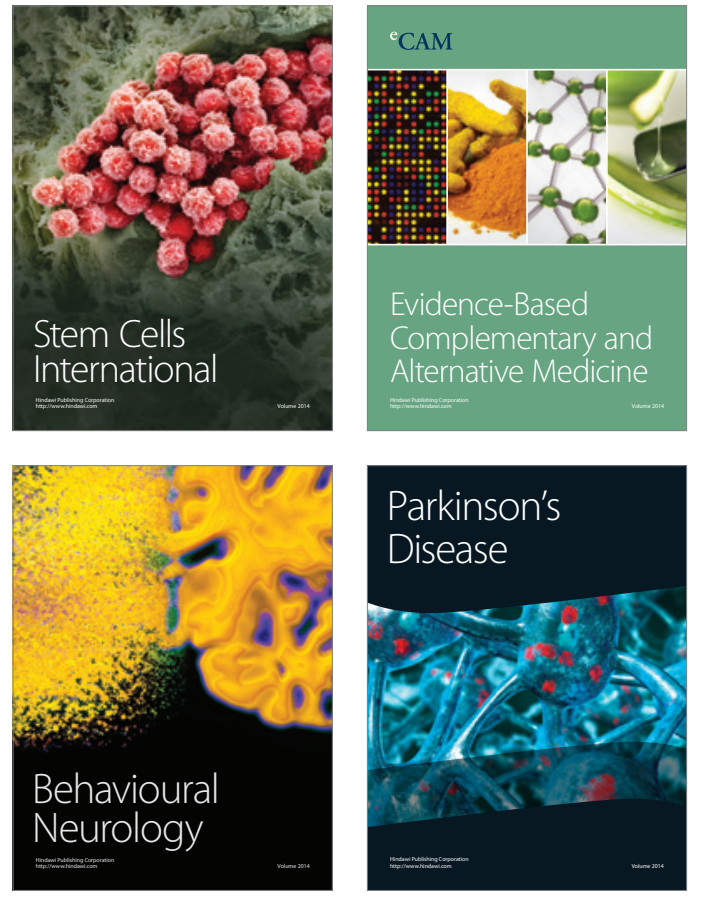
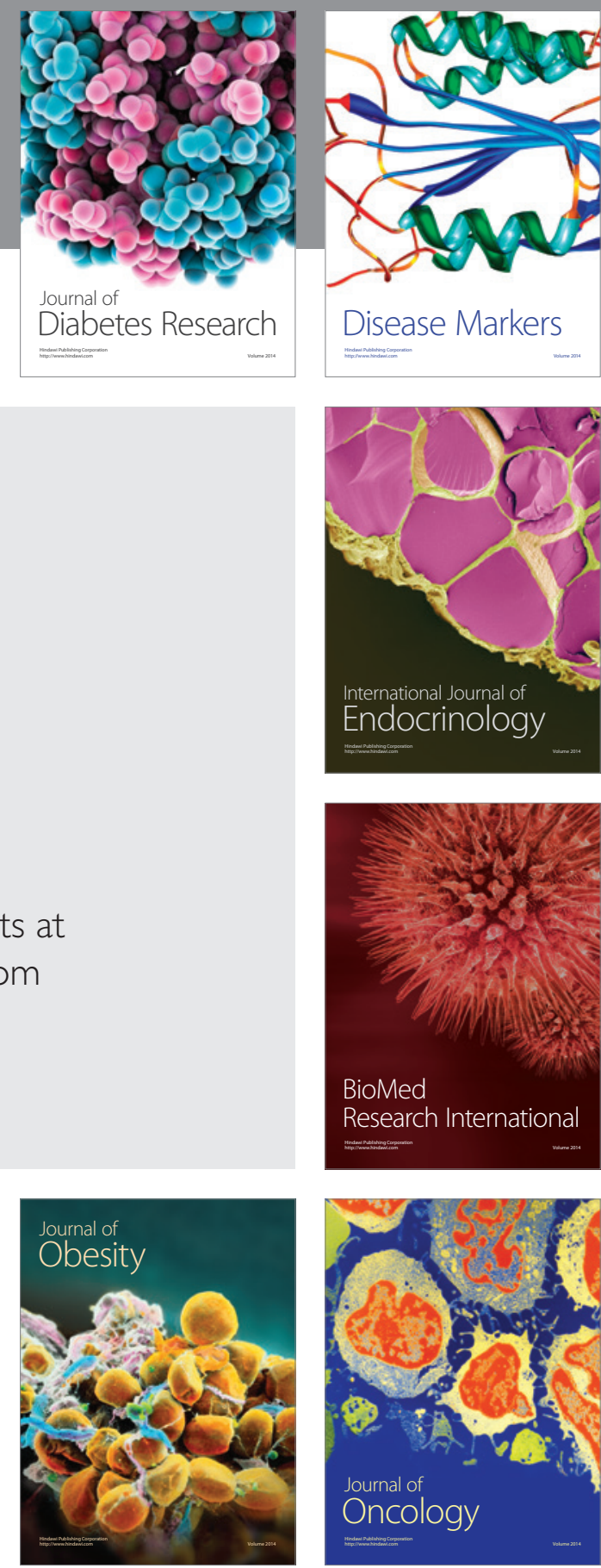

Disease Markers
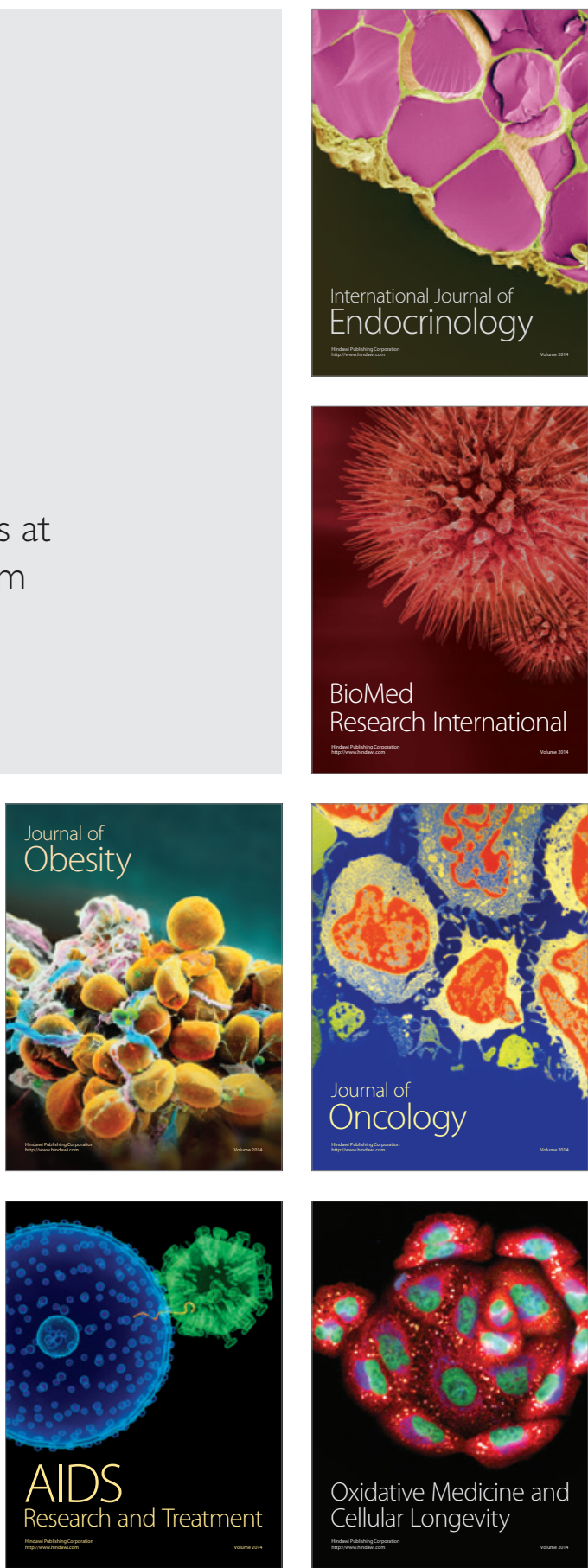